\title{
Emma Bratte, la femme folle du Livre d'Emma
}

Pooja Booluck, Université de Colombie Britannique

Née dans une période troublée par les guerres mondiales et le colonialisme, la littérature africaine et antillaise traite aujourd'hui de sujets tels que la violence, l'oppression des femmes et la résistance de celles-ci face à cette oppression. On se demande si être à la fois noire et folle n'est pas considéré comme une construction dans cette branche de la littérature contemporaine (Epupa dans Les aubes écarlates de Léonora Miano ; Télumée dans Pluie et vent sur Télumée Miracle de Simone Schwartz-Bart). Certaines voix narratives caractérisent même cette condition comme étant une condamnation au malheur : « tu dois te dire qu'être Négresse et folle, c'est le comble de la malédiction »(Agnant 26). Dans Le livre d'Emma, Marie-Célie Agnant nous présente Emma Bratte, jeune femme haïtienne qui tente d'expliquer la raison pour laquelle elle a tué sa petite fille. Elle refuse de fournir ces informations au personnel blanc de l'hôpital psychiatrique dans lequel elle est admise, car elle tient à s'exprimer dans sa langue maternelle. Frustré dans ses efforts pour tirer de sa patiente l'information qu'il cherche, le psychiatre, Dr. MacLeod, invite Flore, une interprète antillaise, à déchiffrer les paroles et la mémoire de cette « criminelle ». À travers le discours oral d'Emma, Flore cherche à comprendre les souffrances de cette femme ainsi que les motifs du meurtre de sa fille, Lola.

Comme c'est le cas du personnage de Mireille dans Un chant écarlate de Mariama Bâ et de celui de Louise dans Chanson douce de Leila Slimani, on constate que la littérature décrit souvent les femmes criminelles comme des femmes qui dérangent et interrogent les normes de genre et les tabous sociaux (Chevillot et Trout 268). En effet, le crime d'Emma, chez Agnant, dérange la norme sociétale : comment une femme peut-elle tuer son enfant ? La société ne pouvant comprendre la raison principale de ce crime inhumain, considère Emma comme étant folle. Cette étude s'intéresse à la manière dont la littérature antillaise redéfinit la notion de folie sans s'appuyer seulement sur une mentalité colonialiste ou patriarcale. Il convient pour cela de se demander quelles sont les raisons principales pour lesquelles Emma a coupé sa lignée générationnelle en tuant sa progéniture. Est-ce tout simplement la cruauté de cette femme, sa condition mentale, ou une décision calculée en fonction de la réalité étouffante du passé ? Quel rôle le silence joue-t-il dans l'explication (ou la non-explication) du crime ? À travers ces questions, cet article nous 
permettra d'exposer et de réévaluer les présupposés au sujet d'une femme haïtienne que l'on n'arrive pas à comprendre sans avoir recours à la notion de folie.

\section{La folie d'Emma Bratte}

Avant de comprendre la cause du meurtre de Lola, il est important de s'attarder sur la définition de la folie. Marcelo Otero affirme que, même aujourd'hui, «aucune définition ne spécifie de façon adéquate les limites précises du concept de trouble mental» (35). Cette interprétation écarte d'emblée toute définition consensuelle de la folie, sur laquelle s'entendrait par exemple la collectivité scientifique, qui y verrait une maladie associée à des individus psychologiquement perturbés et socialement problématiques $(1 ; 35)$. Pourquoi donc le docteur MacLeod conclut-il si vite qu'Emma est folle ? Pour répondre à cette question, Aurélie Marguerite propose de « revenir sur l'évolution du fou en société, ou plutôt l'évolution de la société à l'égard du fou » (5). Dans Histoire de la folie à l'âge classique, Michel Foucault explique qu'au Moyen Âge, les victimes de la lèpre étaient mises à l'écart de la société pour le bien des autres (19). Marguerite, suivant Foucault, explique que «les épidémies de lèpre, difficilement soignées et maitrisées à l'époque, ont mené à l'ouverture de nombreuses léproseries qui, quelques décennies plus tard, seront reconverties en lieux d'accueil pour les fous » (5). Peu à peu, cet isolement des individus a donné lieu à une forme d'institution qui bannit les individus qui sont, d'une manière ou d'une autre, repoussés par la société. De la même façon que le malade est rejeté et enfermé, le fou est écarté de la sphère sociale, car il ne peut plus répondre aux normes. D'une certaine manière, la folie produit un être humain qui est déjà mort, car il est déchu de son rôle dans la société. Il va sans dire que la criminelle souffre du même destin que la folle. Fanny Mahy utilise le terme «monstre » comme métaphore pour décrire le criminel : «la métaphore du monstre [...] désigne [des personnes] au psychisme malade et déviant ou au comportement non conforme avec l'idéal social de la morale » (159). On se demande si on considère la criminelle comme étant folle, car pareillement à une femme folle, et si la criminelle est vue «avec l'excès, l'écart, la norme et le franchissement des limites tracées par la société » (159).

Pareillement, Emma, repoussée par une société qui refuse de comprendre le motif derrière le crime qu'elle a commis, est enfermée dans un hôpital psychiatrique. Au cours du roman, on voit que cette femme a été rejetée depuis son enfance. Antoinette Marie Sol explique qu' ' [Emma] est hantée par un sentiment de non existence depuis son enfance, par le rejet de sa mère, par le néant 
où est tombée l'histoire de sa race, par le refus de son mémoire par le jury académique et, par [conséquent], de sa mémoire » (979). Le lecteur constate qu'Emma se perçoit déjà condamnée à ne plus vivre en société à cause de ce manque d'appartenance qui émane de son enfance. Comme un fou ou un malade, elle est mise de côté, même par sa mère, à cause de la couleur de sa peau : « je sais déjà que Fifie me voue une haine incommensurable [...] je l'ai senti sitôt que j'ai mis le nez dehors » (Agnant 62). D'où vient cette haine qui entoure cette femme noire ? Peut-on vraiment dire que Fifie détestait sa propre fille ? Pendant son séjour avec Mattie, sa parente, elle comprend que ce sentiment vient de loin. Comme celle-ci le lui explique, ce mal qu'éprouve sa mère touche beaucoup de femmes noires : «Il coule dans nos veines, nous l'ingurgitons dès la première gorgée du lait maternel » (121). Peu à peu, Emma comprend la raison pour laquelle sa mère et sa tante la traitent d'une manière aussi inhumaine.

Cette condition dont souffrent les femmes noires, selon Mattie, va au-delà de l'enfance. Les thèmes de rejet et de folie caractérisent chaque phase de la vie d'Emma. Alors qu'elle voulait rendre hommage à ses aïeules et dévoiler le non-dit de l'Histoire, cette tentative est piétinée par le refus de sa thèse. En donnant comme explication que sa thèse manque de cohérence, le jury implique que cette doctorante ne sait pas ce qu'elle raconte. L'écriture d'Emma revêt toutefois une certaine importance pour son peuple : « $[S]$ a thèse représente une tentative formelle de corriger non pas les crimes du passé, mais leur représentation. Elle voulait insérer sa voix dans le champ discursif traitant de l'esclavage et dont les limites se trouvent loin des crimes et loin des victimes » (Boucher 100). À travers sa tentative d'écrire pour son peuple, elle dévoile une partie de l'Histoire qui a été réduite au silence. Emma prétend qu'à travers son écriture, ses semblables retrouvent leur voix. Par exemple, après avoir transmis à Flore tout ce que Mattie lui avait appris sur l'histoire de leurs ancêtres, Emma emploie explicitement un adjectif possessif collectif : « Notre histoire est écrite avec du sang » (132). En revanche, sa thèse n'est pas acceptée car elle, femme noire, ose remettre en question ce qu'ont écrit les Blancs. Elle, descendante d'esclave, ose corriger l'Histoire telle qu'écrite par l'Occident. Si elle avait été acceptée, cette thèse aurait pu servir de mémoire utopique où « une image d'un état idéal, où tous les maux et les torts de la société présente sont guéris et redressés » (Messac 183). S'il pourrait paraitre trop optimiste d'assumer que le travail d'Emma représente « un état idéal » ou qu'il puisse guérir tous les maux de la société antillaise, sa démarche académique apparaît non moins comme un moyen de diffuser la voix des opprimées et de réaliser le rêve symbolique qu'elle avait déjà fait comme enfant : «J'ai rêvé une fois que notre 
savoir, à nous les Négresses, se transformait en lait, en bon lait mousseux, en bon lait tout bleu à force d'être blanc, et nous le donnions à téter à tous » (33).

Dans quelques textes antillais tels que Morne câpresse de Gisèle Pineau et Ti Jean l'horizon de Schwartz-Bart, l'esclavage et la condition noire contribuent à peindre une image dystopique, alors que chez Agnant, des personnages tels qu'Emma essaient de créer un espace plus optimiste. Bill Ashcroft explique que

African visions of the future are driven by the dynamic of hope, which took shape in the experience of colonisation, but continues through an anticipatory consciousness [...] Utopia is necessary because it is only from the no-place of a utopian future that ideology - particularly the teleological ideology of history - can be contested. What remains remarkable about African literature and cultural production is the stunning tenacity of its hope and its grounded vision of possibility. (113)

On comprend que la thèse qu'Emma a écrite parle d'espoir et de possibilités, ce qui tend à changer la façon dont le monde perçoit l'Histoire. Elle cherche à y créer un espace nouveau dans lequel le monde perçoit sa race d'un angle autre que celui du colonisateur. En revanche, le lecteur constate que cet espace reste toujours le «non-lieu» dont parle Ashcroft : le patriarcat et l'Occident (le jury académique et le médecin) refusent de voir autre chose qu'un «manque de cohérence » (73) dans tout ce qu'elle dit et écrit.

En explorant les horreurs du passé, Emma devient opaque pour ceux qui ne sont pas victimes de cette mémoire traumatique. Le docteur MacLeod est décrit comme « un petit homme courtaud, aux traits effacés [qui] s'adressait aux gens avec une sorte de calme qui conférait à son visage l'immobilité froide de la pierre » (Agnant 9). Les mots « traits effacés » et la comparaison de son visage à «l'immobilité froide de la pierre » montrent le paternalisme et l'autorité de cet homme. Étant blanc, homme et médecin, il refuse d'être questionné et « ne cherche pas forcément à savoir si la patiente est atteinte de folie ou non, mais plutôt à confirmer un diagnostic de la folie déjà établi » (Marguerite 17). Le lecteur se rend compte que, tout comme le jury, le médecin refuse de comprendre le discours d'une femme antillaise qui remet en question la prétendue supériorité de la culture qu'il représente. Il se conforme davantage au modèle traditionnel du monde patriarcal et blanc de la psychiatrie, «monde qui atteint la réintégration du patient par le biais d'un échange entre le patient et le thérapeute dans lequel le premier explique et ordonne l'expérience pour le second au cours d'une relation hiérarchique de domination » (Sol 979). Ayant déjà établi un diagnostic, il refuse d'entendre la voix de sa patiente, car après tout, ce qu'il explique et ordonne 
pèse de beaucoup plus de poids que ce qu'Emma peut transmettre dans cet «échange ». Le discours d'Emma est caractérisé comme étant incompréhensible et incohérent car il est conçu par « une mentalité de colonisateur [à la fois] paternaliste, irrité et condescendant » (AdamowiczHariaz 158).

À travers sa parole et son écriture, Emma dérange la norme de ce qui a été dit depuis longtemps par les Blancs. D’une certaine manière, elle est différente des autres de la même façon que le fou est défini par la différence. Monique Plaza définit « la folie comme un rapport entre différentes forces sociales plutôt qu'une maladie mentale»(9). Il est évident que le discours d'Emma n'appartient ni à celui du dominant ni à celui du patriarcat - il dévoile les injustices subies par les femmes noires et remet en question l'écriture des Blancs. Ce fait est la raison principale pour laquelle Emma refuse de s'exprimer en français. Il se peut qu'elle reconnaisse que le passé se reproduira: que sa parole va être «tronquée, lobotomisée, excisée, mâchée, triturée puis recrachée en un jet informe » (Agnant 24). Elle n'accepte ni de se lier à l'autre, ni de parler la langue de l'autre, car « les bêtes qui hurlent n'empruntent jamais la voix des autres » (16). Elle efface cette notion de différence qui forme la définition de « folie » en se confiant à une semblable. En parlant sa langue maternelle avec Flore, elle change de stratégie pour émettre sa parole. Elle choisit une Antillaise à qui raconter son histoire et à qui dévoiler la raison pour laquelle elle a tué sa fille. Cependant, alors que le choix de parler sa langue maternelle est justifié, l'acte inhumain de tuer sa fille est-il soutenu par des raisons convaincantes ?

\section{La mort de Lola}

Frédérique Chevillot et Colette Trout se demandent si « la femme qui tue est [...] plus monstrueuse que son alter ego masculin parce qu'elle transgresse la notion normalisée et abusive d'une « nature féminine » (12). La société patriarcale dans laquelle vit Emma pénaliserait-elle un homme de la même manière s'il avait commis le même crime ? Emma est perçue comme étant dangereuse car, étant femme, noire et criminelle, elle dérange les normes sociétales qui indiquent que la femme doit être passive et inoffensive. Le fait que cet acte impitoyable ait été exécuté par une femme, par une mère de surcroît, est ce qui hébète encore plus la société dans le roman. Comment expliquer que la responsable de la mort d'un enfant est la personne qui l'a mis au monde ? Sans doute, les femmes criminelles dérangent aussi les stéréotypes liés au genre sexuel féminin : il est impensable qu'une femme, plus précisément qu'une mère, s'adonne à des actions 
criminelles. De plus, Emma étant noire, elle est victime d'une double transgression, car le sujet du stéréotype est aussi lié à la race noire. N'ayant pas trouvé de motif pour expliquer la mort de Lola, la presse a recours aux clichés qui entourent la femme noire : « une noire sacrifie son enfant... une affaire de vaudou ?» (Agnant 16). Les personnages du roman qui personnifient une attitude occidentale, tels que le docteur MacLeod ou le journaliste cité ici, refusent de tenir compte des faits qui se trouvent hors de ce qu'ils ont appris dans les livres sur l'histoire officielle, ce qui explique leur recours à des stéréotypes pareils.

Selon les quotidiens, l'état dans lequel se trouve Lola quand elle est retrouvée morte est abominable, «son corps menu, déchiqueté, s’est retrouvé dans les boites à ordure de toutes les demeures, parmi les papiers sales, les bouts de chiffon et les détritus emportés par les éboueurs » (16). Pour quelle raison Emma a-t-elle tué son enfant d'une manière aussi odieuse ? Est-ce tout simplement la condition mentale de cette femme ou alors le résultat d'une souffrance intergénérationnelle insoutenable ? Emma s'associe tant à cette mémoire et à ces injustices du passé qu'elle développe une sorte d'obsession, ce qui l'empêche de mener une vie dans le présent. Chaque personne à qui Flore s'adresse pour obtenir plus d'information, parle de l'obsession Emma au sujet du passé : la travailleuse sociale le démontre quand elle se souvient de ses séances avec elle : «[Emma] avait chez elle une petite valise de cuir fripée, remplie de papiers pour cette thèse qu'elle n'en finissait pas de réécrire » (14). De plus, le partenaire d'Emma, Nicholas Zankoffi, explique que «par son refus d'enterrer ses morts, Emma voulait se détruire ; elle vivait dans leur ombre, proie de leurs fantômes » (48).

Suite à ce qu'elle a trouvé au sujet de l'esclavage pendant sa recherche et de ce qu'elle a appris de Mattie, Emma s'identifie pleinement avec ses aïeules. Mattie lui transmet son histoire personnelle, collective, familiale. À travers l'histoire de Rosa, de Baptiste, de Fifie, de Cécile, de «l'histoire d'une île, [du] lambeau de l'époque coloniale, [du] vestige de sa cruauté et de son inhumanité », la «folie » du personnage principal se manifeste (17). Obsédée par le désir de changer la représentation que l'on se fait de sa lignée, Emma tente de faire entendre le non-dit de l'Histoire. Alors que sa voix a été étouffée par l'Occident, elle se confie à Flore, une semblable.

Au cours de leur échange, Emma explique à Flore qu'elle voit souvent les femmes des anciennes générations. Elle voudrait apprendre davantage de celles-ci, mais depuis son enfermement dans l'hôpital psychiatrique, elle les perd : «il m'arrive d'appeler à mon secours Kilima, Cécile, Emma avant moi, puis Rosa, ces marronnes éternelles. J'implore leur mémoire, 
elles sauront me guider, je me dis, mais je ne les vois point [...] depuis que je suis enfermée ici, [j]e ne rêve plus du tout » (174). Les rêves d'Emma sont aussi marquants : ils symbolisent le fil d'une génération à l'autre, l'espoir, ils sont des voix qui lui parlent et qui la guident dans la formation de ses connaissances et de sa conscience historique. Le roman crée l'impression qu'Emma représente plusieurs voix : elle est la seule qui survit, elle « crie pour ses sœurs mortesnées » (Curtis 70), elle vient d'une « colonie de morts vivants » (Agnant 27) et elle écrit une thèse pour honorer ces voix. Les rêves qu'elle faisait quand elle était petite préfigurent ce rapport avec le passé, alors qu'elle détruisait tout et n'avait rien à quoi s'accrocher. L'on comprend que ses ancêtres lui montrent que le passé a toujours un effet sur le présent. On voit qu'elle détruit non seulement ses relations avec le monde dans lequel elle vit, mais aussi avec sa fille. Quand elle cesse de rêver, elle perd ce lien onirique entre elle et ses aïeules, ce qui explique pourquoi elle cesse de lutter.

Le livre d'Emma traite ainsi non seulement d'infanticide, mais aussi de suicide. Emma prend à cœur ce que dit Mattie lorsque celle-ci lui explique qu'il n'y aura plus d'autre génération après la sienne : « tu es la dernière de la lignée $[\ldots]$ après toi, ne viendra personne » (152). Les paroles de Mattie à Emma renvoient à cette souffrance qu'elles ont toutes subie, les femmes noires ayant historiquement fait l'objet d'une indéniable oppression. Malgré qu'Emma reste engagée dans sa lutte contre les forces oppressives de l'Histoire, on constate qu'afin de ne plus laisser souffrir les générations à venir, elle tue sa progéniture et se retire complétement de l'Histoire, qui piétine les femmes noires. Catherine Lord explique que «l'infanticide et le suicide peuvent être perçus comme une façon, pour Emma, de s'extraire de l'histoire, qu'elle considère comme une éternelle répétition. En mettant fin à la vie de sa fille et la sienne, elle effectue une double interruption de la filiation réelle ; elle ne sera ni la fille ni la mère de quiconque et ainsi, aucune des souffrances héritées des ancêtres ne sera perpétuée » (60). Emma épargne à sa fille la dure réalité que ses aïeules et elle-même ont vécue. En l'éliminant, elle rompt ce legs difficile afin que Lola ne soit pas définie par ce traumatisme intergénérationnel. Sol constate qu'Emma, «qui s'identifie avec ces 'marronnes éternelles' répète le geste des 'femmes du clan' comme l'a prédit Mattie. Emma, comme Kilima, tuera sa fille sans excuses, sans explication en disant seulement qu'elle « devait mourir » (162), puis se suicidera une fois son (ses) histoire(s) transmise(s) suivant le destin d'autres femmes du clan (125) » (981). D'une certaine manière, l'histoire de ces femmes se répète - Emma tue sa fille de la même façon que Kilima a essayé de tuer la sienne, avant de se 
suicider (Agnant 173). Pour Emma et Kilima, il s'agit de rompre le cycle de la « malédiction » qui consiste à être une femme noire dans un monde irrémédiablement injuste. Telles les victimes de viol qui tueraient leurs enfants, elles sacrifient leur progéniture et livrent un combat intime contre l'ennemi (Préher 5). Pour ces femmes antillaises, l'infanticide et le suicide mettent fin à cette souffrance intergénérationnelle insoutenable.

L'infanticide et le suicide constituent-ils des symboles de la libération? Sol voit « le topos de la mort comme affirmation de liberté et de refus »(970). Dans le contexte historique de l'esclavage, tuer sa progéniture et se suicider est un acte de générosité. Emma épargne à sa fille les horreurs de la société blanche. Il est vrai que ce crime est impardonnable du point de vue des autorités blanches du roman, mais une analyse du passé collectif d'Emma permet de comprendre que la mère voulait protéger son enfant. Comment laisser sa fille souffrir dans cette répétition des actes immoraux qui se passent d'une génération à l'autre ? Ce que fait Emma est un acte d'euthanasie, car elle voit déjà le futur pénible de sa fille et l'en préserve. Ainsi, Mattie lui avait déjà dit : «Il n'est pas étonnant qu'au bout de ce tunnel nous guette la démence, et c'est alors que nous détruisons notre propre chair, parce que nous tremblons pour elle, nous savons ce qui l'attend» (Agnant 120). Frantz Fanon explique que «[le Noir] a implicitement admis la valorisation des différences de fait et l'essentielle blessure qu'elle maintient au cœur de ce monde. C'est alors qu'il adopte une attitude tout entièrement négative » (19). Cette attitude négative englobe la réalité de toutes les femmes noires du roman qui savent qu'elles feront toujours l'objet d'aliénation. De sa part, Homi K Bhabha montre que « infanticide was recognized as an act against the system and at least acknowledged the slavewoman's legal standing in the public sphere » (24). Alors qu'on pourrait dire qu'Emma partage cette notion d'aliénation dont parle Fanon avec ses aïeules, elle évoque aussi une autre tentative de se faire entendre dans la société en tuant sa fille : elle démontre que ce n'est qu'elle qui peut prendre des décisions à propos de sa progéniture.

Après avoir conté l'histoire de Kilima, Emma annonce que « quand tout sera terminé, [son] sang s'en ira rejoindre celui de Kilima, de Rosa, d'Emma avant [elle], et des autres » (153). Pour elle, mourir est un acte de renaissance, car à travers la mort, elle rejoindra ses aïeules. Elle pourra enfin être parmi les siennes, parmi des femmes qui comprendront sa souffrance, et dans un endroit où sa parole ne sera plus jamais étouffée. Son suicide à la fin du roman explique pourquoi elle n'arrêtait pas de parler de son départ de ce monde : «elle disait toujours, elle disait sans cesse qu'elle reprendrait un jour la route des grands bateaux » (180). Somme toute, l'infanticide et le 
suicide dans Le livre d'Emma sont justifiés par la tentative de la part du personnage principal d'empêcher le cycle de la malédiction et de l'injustice.

\section{Le silence des « Négresses »}

Scott Lyngaas propose une autre explication pour le meurtre de Lola : «after her thesis was rejected for the second time, Emma murdered her daughter, it seems, to save her from the silence that was certain to surround her, to save her from the miserable existence that she and her ancestors have experienced » (972). De quel silence parle Lyngaas ? Est-ce un silence imposé ou volontaire? Ne s'applique-t-il qu'aux «négresses »? Fatma Agoun Perpère explique que « la négresse est [...] un être hors classe, généralement méprisé, dont la pigmentation marque de façon indélébile la servitude ou l'ascendance servile et passe le plus souvent pour la manifestation de la couleur de son âme » (81-82). Cette citation met en lumière le traitement que reçoit Emma : étant noire, elle est censée, selon la logique que décrit Perpère, être inférieure aux autres races et soumise à la servitude. Cela explique aussi la consternation qu'éprouve la communauté blanche dans laquelle elle se trouve, car selon les normes établies, une femme noire n'est pas capable de prendre ses propres décisions ni de comprendre elle-même sa place dans l'histoire.

Lors de ses séances avec Flore, Emma lui dit de « [ne jamais oublier qu'une] femme qui parle, crie et hurle en vain fait autant de bruit qu'un nuage. Mieux vaut avaler sa langue, croismoi, comme le faisaient nos grands-mères sur les bateaux » (Agnant 119). Cette comparaison du cri d'une femme à un nuage montre que la femme noire peut crier, parler pour essayer de se faire entendre, mais le monde la piétinera toujours. La citation de Lyngaas suggère qu'en tuant sa fille, Emma la protège de la douleur qui vient de ce fait d'être piétinée. Elle préfère l'ôter de ce monde, un monde qui va lui couper la parole et qui lui imposera une histoire qui n'est pas la sienne. Quand Flore lui demande de décrire Lola, elle répond qu'elle ressemblait au vent (95). Alors que le nuage est silencieux, le vent a la propriété d'être fort, bruyant et libre. Lesley Curtis explique que la mort de Lola «empêche sa réduction au transparent; et même si c'est sa mère qui choisit [sa mort], Lola restera toujours incompréhensible à ceux qui ont refusé l'histoire de sa mère » (75). Tout comme Emma, l'Occident va refuser d'entendre la parole de Lola, car dans le roman, les Blancs refusent de comprendre que le passé colonial a toujours un effet traumatique sur les Noirs.

Il semble que beaucoup de personnages noirs dans la littérature antillaise choisissent paradoxalement de garder le silence justement à cause de ce silence imposé. Dans Histoire de la 
femme cannibale de Maryse Condé, Fiéla, femme antillaise qui tue son mari parce qu'il la trompait, refuse de dire quoi que ce soit à la presse et aux policiers. Tout comme Emma, elle comprend que sa parole ne mènera à rien, que si son histoire ne ressemble pas aux histoires déjà écrites, déjà établies par les autorités blanches, la sienne sera opaque. En revanche, le personnage principal, Rosélie, également antillaise, comprend les motifs derrière le meurtre, car elle se reconnaît en Fiéla. D’une certaine manière, Flore et Rosélie agissent comme intermédiaires pour Emma et Fiéla. Il se peut que la fin des deux récits n'apporte pas de grand changement dans la vie des personnages principaux, mais les auteures de ces deux romans livrent un message semblable au lecteur : leurs histoires remettent en question la pensée coloniale et tout ce qui a été écrit par les colonisateurs. Awa Thiam, une Africaine, se met de même à l'écoute de ses sœurs africaines dans son œuvre intitulé Parole aux Négresses: «Longtemps les Négresses se sont tues. Il est temps qu'elles redécouvrent leurs voix, qu'elles prennent ou reprennent la parole, ne serait-ce que pour dire qu'elles existent, qu'elles sont des êtres humains et qu'en tant que tels, elles ont droit à la liberté, au respect, à la dignité » (17). Si, à un moment donné, certaines femmes noires se taisaient, d'autres, comme Thiam, se mettent debout contre les forces qui les oppriment. Il se peut que certains Blancs ne les écoutent pas quand elles parlent, mais en écrivant des textes comme le fait Flore suite à sa conversation avec Emma, leurs voix se font entendre. Par conséquent, le silence dans Le livre d'Emma se conçoit comme une stratégie de défense : Emma refuse de divulguer quoi que ce soit au médecin blanc, car ce dernier utiliserait le discours de celle-ci pour valider ses propres préjugés.

Que peut-on dire du livre qu'écrit Flore après la mort d'Emma? Alors qu'Emma est enfermée dans cet hôpital, accablée et vaincue à cause de son incapacité à se faire entendre, Flore deviendra sa parole. Le livre d'Emma résulte d'une tentative de la part de Flore de briser ce silence qui entoure les femmes noires. Alors que Flore ne peut pas contrôler la réception de son livre, il s'agit tout de même d'une tentative de faire comprendre la vision d'Emma. On pourrait dire que ce livre est un lieu de mémoire utopique. Terme formulé par Pierre Nora, un lieu de mémoire peut être un monument pour commémorer un événement - un livre, une thèse, un objet abstrait ou une lettre qui contient un récit. Il ajoute que «memories are crystalized and transmitted from one generation to the next - but [a memory space] is also symbolic, since it characterizes by referring to events or experiences shared by a small minority, a larger group that may not have participated in them » (19). L'écriture de Flore réalisera la vision utopique de ce que la parole d'Emma n'a pas 
pu faire. Elle va raconter l'histoire et la mémoire des générations des femmes noires qui ont résisté à la subjugation. À travers cette écriture, le lecteur va enfin pouvoir reconnaître la souffrance de ces femmes noires - on leur donnera un nom et une place dans l'Histoire.

Le livre de Flore expliquera également la cause de la mort de Lola et la raison pour laquelle on qualifie Emma de folle. Flore y permettra au lecteur de comprendre dans quelle mesure la puissance coloniale a toujours un effet sur le monde qu'elle a colonisé. Curtis ajoute que «c'est aux lectrices et aux lecteurs de reconnaître ce que le système scientifique occidental ne reconnaît pas » (73). De plus, la langue du livre, comme la langue de la thèse, est le français. Le livre est une réécriture de ce qu'Emma a écrit. Les représentants de la communauté scientifique occidentale, tels que le docteur MacLeod, vont enfin entendre la voix de cette femme qui a tant lutté pour s'affirmer.

\section{Conclusion}

Avec Le livre d'Emma, Agnant raconte l'histoire d'une femme noire qui lutte pour faire entendre l'histoire oubliée de ses aïeules. En créant un personnage de femme qui met sa parole au service de la mémoire troublante d'un passé collectif toujours présent, elle pousse son lecteur à réévaluer les préjugés au sujet de la femme dite à la fois « folle » et « criminelle ». Juridiquement parlant, le crime qu'Emma a commis est impardonnable car elle a tué un être humain. En revanche, il est important de relever la raison pour laquelle elle a dû le faire. Obsédée par le passé, elle est obligée de rompre le cycle de l'oppression des femmes noires. Emma commet un acte aussi impitoyable non pas par cruauté, mais par amour pour sa fille. Elle la protège contre l'injustice qui accable depuis longtemps ses aïeules. Suite à ce crime, la société blanche du roman conclut qu'elle est folle sans vraiment l'écouter. Au lieu de faire un rapport complet qui inclut la parole de sa patiente, le docteur MacLeod traite celle-ci en fonction d'un diagnostic déjà établi. Il refuse d'entendre le discours d'une femme noire, car étant blanc et homme, sa parole est automatiquement valorisée. En revanche, on constate que le livre qu'écrit Flore agit comme un lieu de mémoire utopique car il va faire entendre les voix des femmes noires - l'Histoire subie par ce groupe sera reconnue par le monde. Finalement, Emma, femme dite « criminelle » et « folle », ne commet pas ce crime à cause d'une affaire de vaudou, mais plutôt pour se libérer d'une mémoire troublante du passé qui la hante toujours. 


\section{Ouvrages cités}

Adamowicz-Hariasz, Maria. «Le Trauma et le témoignage dans Le Livre d'Emma de Marie-Célie Agnant ». Symposium : A Quarterly Journal in Modern Literatures 64.3 (2010). 158-162. Agnant, Marie-Célie. Le livre d'Emma. Montréal : Remue-Ménage, 2001.

Agoun Perpère, Fatma. «La peau et le masque : Images de la femme noire dans les contes maghrébins ». Cahiers Sens Public 10.2 (2009). 81-96.

Ashcroft, Bill. «African Futures : The Necessity of Utopia». International Journal of African Renaissance Studies : 94-114.

Bâ, Mariama. Un chant écarlate. Dakar : Nouvelles éditions africaines, 1981.

Bhabha, Homi K. The Location of Culture. London : Routledge, 2004.

Boucher, Colette. Paroles et silences chez Marie-Célie Agnant : L'oublieuse mémoire d'Haïti. Paris : Karthala, 2013.

Chevillot, Frédérique et Trout, Colette. Rebelles et criminelles chez les écrivaines d'expression française. Amsterdam : Rodopi, 2013.

Condé, Maryse. Histoire de la femme cannibale. Paris : Mercure de France, 2003.

Curtis, Lesley S. «'Vite elle se referme' : L'opacité dans Le livre d'Emma de Marie-Célie Agnant ». Women in French Studies 21 (2013). 68-78.

Fanon, Frantz. Peau Noire, Masques Blancs. Paris : Seuil, 1952.

Foucault, Michel. Histoire de la folie à l'âge classique. Paris : Gallimard, 1972.

Lord, Catherine. «La récupération identitaire dans Le ciel de Bay City de Mavrikakis et Le livre d'Emma d'Agnant », mémoire de maîtrise, Université Queen's, 2012.

Lyngaas, Scott. «Confronting Haitian History in the Novels of Marie-Célie Agnant ». French Review 84 (2011). 966-977.

Mahy, Fanny. « Le fait divers criminel dans la littérature contemporaine française (1990-2012) », thèse de doctorat, Université Western Ontario, 2014

Marguerite, Aurélie. «La sclérose d'un malaise identitaire : étude comparée de la représentation de la folie dans Le livre d'Emma de Marie-Célie Agnant et dans L'hiver de pluie de Lise Tremblay », mémoire de maitrise, Université de Colombie Britannique, 2014.

Messac, R. Les premières utopies. Audenge : Nihilo, 2009.

Miano, Léonora. Les aubes écarlates. Paris : Plon, 2011. 
Nora, Pierre. «Between Memory and History : 'Les Lieux de Mémoire' ». Representations 26 (1989). 18-20.

Otero, Marcelo. «Le fou social et le fou mental : amalgames théoriques, synthèses empiriques et rencontres institutionnelles ». Sociologies (2010). http://sociologies.revues.org/index3268.html/.

Plaza, Monique. Ecriture et folie. Paris : PUF, 1986.

Préher, Gérard. Les mères et la mort : Réalités et représentations. Bordeaux : PU de Bordeaux, 2008.

Thiam, Awa. La parole aux Négresses. Paris : Denoël-Gonthier, 1978.

Schwarz-Bart, Simone. Pluie et vent sur Télumée Miracle. Paris : Seuil, 1972.

Slimani, Leïla. Chanson douce. Paris : Gallimard, 2016.

Sol, Antoinette M. « Histoire(s) et traumatisme(s) : L'infanticide dans le roman féminin antillais ». French Review 81.5 (2008). 967-984. 